\title{
Evaluation of the Biocompatibility of a Bilayer Chitosan Skin Regenerating Template, Human Skin Allograft, and Integra Implants in Rats
}

\author{
Shah Jumaat Mohd Yussof,, ${ }^{1,2}$ Ahmad Sukari Halim, ${ }^{1}$ \\ Arman Zaharil Mat Saad, ${ }^{1}$ and Hasnan Jaafar ${ }^{3}$ \\ ${ }^{1}$ Reconstructive Sciences Unit, School of Medical Sciences, Universiti Sains Malaysia, Kubang Kerian, 16150 Kelantan, Malaysia \\ ${ }^{2}$ Faculty of Medicine, Universiti Teknologi MARA, Shah Alam, 40450 Selangor, Malaysia \\ ${ }^{3}$ Department of Pathology, School of Medical Sciences, Universiti Sains Malaysia, Kubang Kerian, 16150 Kelantan, Malaysia
}

Correspondence should be addressed to Shah Jumaat Mohd Yussof, shahjumaat@gmail.com

Received 7 April 2011; Accepted 27 May 2011

Academic Editors: M. Afzaal and J. Zhang

Copyright (C) 2011 Shah Jumaat Mohd Yussof et al. This is an open access article distributed under the Creative Commons Attribution License, which permits unrestricted use, distribution, and reproduction in any medium, provided the original work is properly cited.

\begin{abstract}
Introduction. Chitosan is a nontoxic, biocompatible, and biodegradable polymer obtained from chitin by $\mathrm{N}$-deacetylation using strong alkali. Chitosan in a form of a bilayer skin regenerating template can act as a scaffold for regeneration. Integra is a two-layer skin regeneration system, constructed of a matrix of crosslinked fibers that acts as a scaffold for regenerating dermal skin cells. Human skin allografts (HSAs) are the "gold standard" for temporary coverage of clean burn wounds. Objectives. The objective of this study was to conduct in-vivo, preclinical biocompatibility evaluations of Integra, HSA, and Chitosan skin regenerating template (SRT). Methods. Paravertebral subcutaneous pockets were created for the implantation of test materials. Implants were retrieved after 4, 7, 14, 21, and 28 days. Slides of sections through the implants were examined to determine biocompatibility. Results. Chitosan SRT and Integra showed similar inflammatory patterns. HSA showed a higher inflammatory reaction initially which then reduced to levels similar to Integra and Chitosan SRT. Chitosan SRT and Integra also shared similar angiogenesis levels. Towards the end, all implants were degraded with decreased tissue response. Conclusion. Integra, Chitosan SRT, and HSA have been shown to be biocompatible. Integra and Chitosan SRT seem to illicit similar tissue responses.
\end{abstract}

\section{Introduction}

The use of skin substitutes to facilitate early burn wound closure or to treat chronic wounds has been an enticing goal of basic research for decades. However, even through numerous products have been introduced to the wound care market, there is no commonly accepted product that exhibits all of the necessary properties. A skin substitute needs to match the function of natural skin while inducing a minimal inflammatory response.

Wound closure requires a material that restores the epidermal barrier function and becomes incorporated into the healing wound [1]. The material should also create an improved environment for epidermal regeneration and provide a barrier against infection and water loss [2]. The criteria for an ideal skin substitute are that its functional and structural characteristics should closely match autograft skin [3]. It should also be flexible, lightweight, odor-free, and impermeable to microorganisms. The material should be sufficiently permeable to water vapor and allow exudates to leave the wound. It would also be an advantage for the material to be haemostatic, transparent, and biodegradable as it restores normal function to the skin. To date, no substitute or replacement for the patient's own skin has been prepared that approaches the qualities that autogenous material possesses.

Chitin is a naturally occurring and abundantly available polysaccharide obtained from the exoskeleton of crustaceans. It is a nontoxic, biocompatible, and biodegradable polymer. Chitosan also has the ability to form films and is thus used in the formulation of film dosage forms or drug delivery 
systems. It also has hemostatic activity and has been proposed for use as a topical agent in tissue repair [4]. Chitosan has also been shown to have antibacterial, haemostatic, fungistatic, antitumoral, and anticholesteremic properties [5-11]. Chitosan is a popular choice as a tissue support material due to the multiple ways by which its biological, physical, and chemical properties can be controlled and engineered under mild conditions [12]. In this study, the chitosan used is in a form of a bilayer skin regenerating system analogous to Integra.

Integra artificial skin is currently the most widely accepted synthetic skin substitute to be developed for use in burn patients. It was described originally by Yannas et al. in 1980 [13]. Integra has a bilaminar structure, consisting of crosslinked bovine collagen and glycosaminoglycan, coated on one side with a silicone membrane that provides epidermal function. The pore size has been designed to allow migration of the patient's own endothelial cells and fibroblasts. The collagen dermal replacement layer serves as a matrix for the infiltration of fibroblasts, macrophages, lymphocytes, and capillaries derived from the wound bed. As healing progresses an endogenous collagen matrix is deposited by fibroblasts; simultaneously, the dermal layer of Integra is degraded. Upon adequate vascularization of the dermal layer and availability of donor autograft tissue, the temporary silicone layer is removed and a thin, meshed layer of epidermal autograft is placed over the "neodermis." Cells from the epidermal autograft grow and form a confluent stratum corneum, thereby closing the wound and reconstituting a functional dermis and epidermis.

Despite its advantages, Integra is relatively expensive and the learning curve is reported to be steep, with high initial failure rates. The advantages of Integra are that it provides improved elasticity and cosmesis compared with an ultra-thin split-skin graft, with reduced donor-site morbidity compared to standard-thickness split-skin grafts [14].

Human skin allograft (HSA) has been used in wound coverage for as long as autogenic skin transplantation has been performed. Following his report on the first autologous skin transplantation in 1869, Reverdin also described allogenic transplantation. In 1882 Menzel described faster healing of a burn wound after coverage with cadaver skin. The first adverse effects from cadaver skin transplantation were also reported in 1882, when smallpox was transmitted from one amputated arm to four patients through skin transplantation [15].

Human skin allograft (HSA) has also been shown to improve and prepare the granulation tissue in the recipient bed [Hackett, 1974]. The investigators found that the wound bed was better prepared for autografting. This was attributed to enhanced fibroblastic and capillary ingrowth [16]. Portions of the allograft dermis became incorporated into recipient tissue by becoming covered by recipient epidermal cells. Allografted frozen skin appeared to desquamate from the recipient skin without causing an intense cellular immune response. Human skin allograft is the gold standard for treatment of massive burn wounds. HSA is inexpensive, easy to apply, and offers a wide variety of benefits. It has stood the test of time and is now used for nonburn wound management.
The aim of this study is to evaluate and compare the biocompatibility of our Chitosan SRT, Integra, and HSA. An animal study was performed on Adult Sprague-Dawley rats using the implantation test. The biocompatibility evaluation of Chitosan SRT, Integra, and HSA is important prior to clinical use of these materials as skin substitutes. The Integra and Chitosan SRT used in this study are in the form of a bilayer tissue regenerating template. This template is designed to allow tissue ingrowth through its scaffold; hence, its biocompatibility is paramount for it to be able to perform its function.

\section{Methods}

2.1. Outline of Experiment Design. In this experimental study, 50 Adult Sprague-Dawley rats were used. They were divided into 5 groups with 10 rats in each group. During the first stage, all animals underwent the same procedure. Four paravertebral subcutaneous pockets were created. Each rat was implanted with the 3 test materials (Integra, human skin allograft, and Chitosan SRT). Each test material occupied an individual subcutaneous pocket.

These implanted test materials were harvested after 5 different time periods (5 groups: 1, 2, 3, 4, and 5) which were day 4 , day 7 , day 14 , day 21 , and day 28 . Ten rats were harvested at each time interval.

Upon harvesting the implants, its tissue response was assessed by histological examination. Degree of inflammation, evidence of angiogenesis, number of granuloma, giant cells, and the degree of infiltration of surrounding tissue into the implant were recorded.

2.2. Skin Substitutes Used. The Chitosan SRT used in this study was manufactured by the SIRIM Advanced Material Research Centre (Kulim, Kedah) (Figures 4 and 5). It consisted of an inner porous sponge layer bonded to an outer film layer. This structure is thought to be suitable for wound coverage and regeneration of new skin. The inner porous sponge layer acts as a template for cells and capillaries to grow, while the outer film layer acts as a barrier to prevent bacterial invasion and to control water loss. The chitosan was pharmaceutical grade with a molecular weight $(\mathrm{Mv})$ of $6 \times 10^{5}$ daltons and $89 \%$ deacetylation. The chitosan was dissolved in $1 \%(\mathrm{v} / \mathrm{v})$ acetic acid to prepare a $2 \%$ $(\mathrm{w} / \mathrm{v})$ chitosan solution. Sodium bicarbonate was used to neutralize the chitosan solution to $\mathrm{pH}$ 6.3. Glycerol (20\%) was added to the chitosan solution as a plasticizer. The chitosan solution was poured in the PTFE mould and dried to obtain a thin film. For the porous membrane fabrication, the chitosan solution was frozen first at temperature of $-25^{\circ} \mathrm{C}$ using a deep freezer (Ilshin Lab. Co. Ltd.) for 24 hours [17]. The chitosan porous membrane and chitosan film were attached to form a bilayer chitosan membrane using a patented technique [18].

Integra is a commercially available artificial skin. It is United States Food and Drug Administration- (FDA-) approved engineered tissue product for burn treatment and reconstructive surgery. It consists of a two-layer skin 
regeneration system. The outer layer is made of a thin silicone film that acts as a temporary "epidermis." The inner layer is constructed of a complex matrix of crosslinked fibers. This porous material acts as a scaffold for regenerating dermal skin cells, which enables the regrowth of a functional dermal layer of skin.

Human skin allograft was purchased from the Euro Skin Bank in Holland. It acts as a biological dressing for wound coverage.

2.3. Animal Study. Adult Sprague-Dawley rats weighing 350 $\pm 10 \mathrm{~g}$ were obtained from the Animal House of Universiti Sains Malaysia, Health campus, Kubang Kerian, Kelantan, Malaysia. The study was approved by the animal ethical committee of Universiti Sains Malaysia, Health Campus, Kubang Kerian, Kelantan, Malaysia. The rats were fed with a standard rat chow and were given access to water ad libitum. Fifty rats were used in this study. The implantation test was performed for the different skin substitutes. Each rat received four paravertebral subcutaneous pockets. The size of the implant inserted was $1 \mathrm{~cm}$ by $1 \mathrm{~cm}$. The three test materials were each inserted into a single pocket, and the fourth pocket was left empty. The implants were harvested at 5 time intervals: after days $4,7,14,21$, and 28 . Ten rats were harvested at each time interval. All specimens were sent for pathological examination by $\mathrm{H} \& \mathrm{E}$ stain for analysis.

2.4. Histopathological Examination. The tissues were cut longitudinally and submitted for tissue processing according to standard protocols. Six histological sections were made from each tissue block. Each section was then stained using Hematoxylin and Eosin (H\&E) and then examined using a light microscope (X400). The number of inflammatory cells, any evidence of angiogenesis, number of granuloma and giant cells, and the degree of infiltration of surrounding tissue were recorded.

The histological specimens were assessed and quantified using a 5-point scale: 0 -cells not observed, 1-low numbers, 2-some cells observed, 3-cell present all over, and 5-relatively high numbers. All specimens was analysed by a single person.

The degree of invagination was scored using a 5-point scale: 0 -no invagination, 1 -focal, 2 -partial $<25 \%$, 3partial 25\%-50\%, 4-partial 50\%-75\%, and 5-complete invagination $100 \%$.

2.5. Quantitative Assessment. All data were consolidated and analyzed using Microsoft Excel 2003 and SPSS 11.0. Numerical data are reported as the mean and standard deviation (SD). Assumptions for parametric tests were checked prior to determining the appropriate statistical test to use. The Kruskal Wallis and Mann-Whitney tests were used to analyze the data.

\section{Results}

Chitosan SRT and Integra showed similar inflammatory patterns at days 4 and 7 (Figure 1). Inflammatory cells

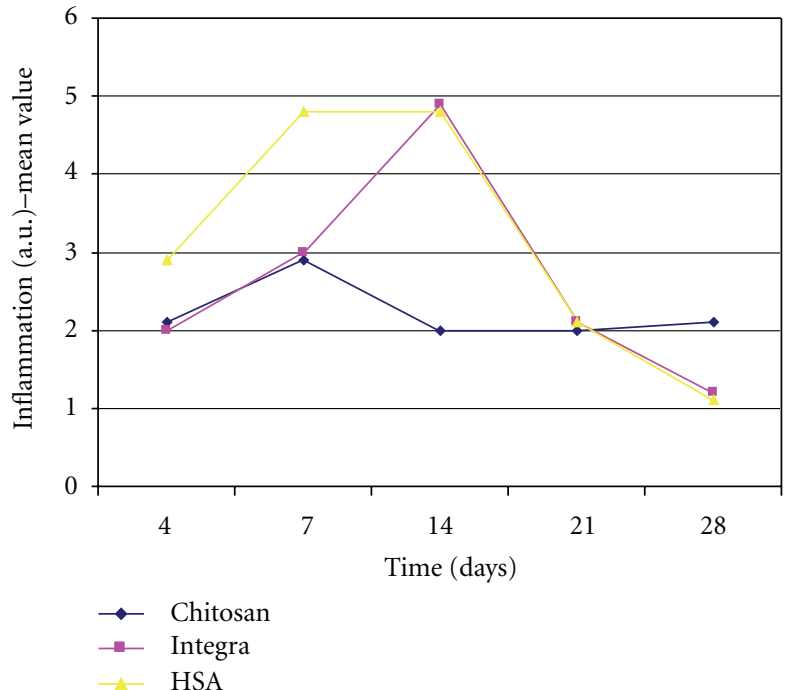

FIGURE 1: The level of inflammation (in arbitrary units) versus time for Chitosan SRT, Integra, and human skin allograft (HSA).

increased from $2.1 \mathrm{au}$ and $2.0 \mathrm{au}$, respectively, at day 4 to $2.9 \mathrm{au}$ and $3.0 \mathrm{au}$, respectively, at day 7 (mean values). However, at day 14 the inflammatory cells in the Integra group increased to $4.9 \mathrm{au}$, compared to the Chitosan SRT samples in which the inflammatory cells decreased to $2.0 \mathrm{au}$. This difference was not statistically significant $(P=0.308)$. By day 21, the inflammation caused by both Chitosan SRT and Integra was the same, 2.0 au and 2.1 au, respectively. By day 28, Integra samples contained minimal inflammatory cells $(1.0 \mathrm{au})$, unlike Chitosan SRT, which demonstrated slightly more inflammatory cells $(2.0 \mathrm{au})$. This difference was not statistically significant $(P=0.308)$ (Tables 1 and $2)$. Chitosan and Integra did not induce any prolonged or exaggerated inflammatory responses throughout the implantation period.

On the other hand, HSA induced a larger inflammatory reaction. At day 4, the level of inflammatory cells was 2.9 au (mean value), and this went up to 4.8 au at days 7 and 14. The level of inflammation then gradually dropped to 2.1 au at day 21 and 1.1 au at day 28. HSA also did not show a prolonged or exaggerated inflammatory response. The differences in inflammatory cell levels between Chitosan SRT and HSA at days $4,7,14$, and 28 were statistically significant $(P<0.001)$ (Tables 1 and 2).

The degree of angiogenesis observed in the Integra, Chitosan SRT, and HSA samples was high (mean $4.9 \mathrm{au}$ ) at day 4. It remained high (4.8 au) in HSA samples up to day 21, before it dropped to 2.1 au at day 28. Chitosan SRT showed a gradual drop in angiogenesis to 4.0 au at day 7 and plateaued at $2.0 \mathrm{au}$ from that point onward. The angiogenesis around Integra samples drops from 4.8 (mean) to 2.1 (mean) by day 7 and remains at this level until day 28 (Figure 2). No statistically significant difference was noted between the 3 skin substitutes at day 4 . At days 7, 14, and 21, there were statistically significant differences between Chitosan SRT and HSA samples $(P<0.005)$ (Tables 1 and 2$)$. 
TABLE 1: Mean score and standard deviation (in brackets) of inflammatory cells, angiogenesis, granuloma, giant cells and tissue invagination upon histological examination.

\begin{tabular}{|c|c|c|c|c|c|}
\hline Day & 4 & 7 & 14 & 21 & 28 \\
\hline \multicolumn{6}{|l|}{ Inflammation } \\
\hline Chitosan SRT & $2.1(0.3)$ & $2.9(0.3)$ & $2.0(0.5)$ & $2.0(0.5)$ & $2.1(0.3)$ \\
\hline Integra & $2.0(0.4)$ & $3.0(0.5)$ & $4.9(0.3)$ & $2.1(0.3)$ & $1.2(0.4)$ \\
\hline HSA & $2.9(0.3)$ & $4.8(0.4)$ & $4.8(0.4)$ & $2.1(0.3)$ & $1.1(0.3)$ \\
\hline$P$ value & $<0.001$ & $<0.001$ & $<0.001$ & 0.799 & $<0.001$ \\
\hline \multicolumn{6}{|l|}{ Angiogenesis } \\
\hline Chitosan SRT & $4.9(0.3)$ & $4.0(0.5)$ & $2.2(0.4)$ & $2.1(0.3)$ & $2.1(0.3)$ \\
\hline Integra & $4.9(0.3)$ & $2.1(0.3)$ & $2.2(0.4)$ & $2.1(0.3)$ & $2.1(0.3)$ \\
\hline HAS & $4.9(0.3)$ & $4.8(0.4)$ & $4.8(0.4)$ & $4.8(0.4)$ & $2.1(0.3)$ \\
\hline$P$ value & $>0.995$ & $<0.001$ & $<0.001$ & $<0.001$ & $>0.995$ \\
\hline \multicolumn{6}{|l|}{ Granuloma } \\
\hline Chitosan SRT & $0.1(0.3)$ & $1.1(0.3)$ & $1.1(0.3)$ & $1.1(0.3)$ & $1.9(0.3)$ \\
\hline Integra & $0.1(0.3)$ & $0.9(0.3)$ & $0.9(0.3)$ & $1.9(0.3)$ & $1.1(0.3)$ \\
\hline HSA & $1.0(0.00)$ & $0.9(0.3)$ & $1.1(0.3)$ & $0.9(0.3)$ & $0.1(0.3)$ \\
\hline$P$ value & $<0.001$ & 0.275 & 0.275 & $<0.001$ & $<0.001$ \\
\hline \multicolumn{6}{|l|}{ Giant cells } \\
\hline Chitosan SRT & $0.9(0.3)$ & $0.9(0.3)$ & $1.1(0.3)$ & $1.9(0.3)$ & $1.1(0.3)$ \\
\hline Integra & $0.1(0.3)$ & $0.1(0.3)$ & $0.1(0.3)$ & $0.1(0.3)$ & $0.1(0.3)$ \\
\hline HAS & $0.1(0.3)$ & $0.1(0.3)$ & $0.1(0.3)$ & $0.1(0.3)$ & $0.1(0.3)$ \\
\hline$P$ value & $<0.001$ & $<0.001$ & $<0.001$ & $<0.001$ & $<0.001$ \\
\hline \multicolumn{6}{|c|}{ Tissue invagination } \\
\hline Chitosan SRT & $4.9(0.3)$ & $4.9(0.3)$ & $4.8(0.4)$ & $4.9(0.3)$ & $4.9(0.3)$ \\
\hline Integra & $4.9(0.3)$ & $4.8(0.4)$ & $4.9(0.3)$ & $4.8(0.4)$ & $4.8(0.4)$ \\
\hline HSA & $0.1(0.3)$ & $1.1(0.3)$ & $2.9(0.3)$ & $2.8(2.0)$ & $4.9(0.3)$ \\
\hline$P$ value & $<0.001$ & $<0.001$ & $<0.001$ & 0.757 & $<0.001$ \\
\hline
\end{tabular}

TABLE 2: Mann-Whitney nonparametric assessment.

\begin{tabular}{|c|c|c|c|c|c|c|c|}
\hline & Skin sub & tes & $\begin{array}{l}P \text { value } \\
\text { (day } 4)\end{array}$ & $\begin{array}{l}P \text { value } \\
(\text { day } 7)\end{array}$ & $\begin{array}{l}P \text { value } \\
(\text { day } 14)\end{array}$ & $\begin{array}{l}P \text { value } \\
(\text { day } 21)\end{array}$ & $\begin{array}{l}P \text { value } \\
\text { (day 28) }\end{array}$ \\
\hline \multirow{2}{*}{ Inflammation } & Chitosan SRT & Integra & 0.308 & 1.000 & 0.308 & N/A & 0.308 \\
\hline & & HSA & 0.003 & 0.292 & 0.003 & N/A & 0.003 \\
\hline \multirow{2}{*}{ Granuloma } & Chitosan SRT & Integra & 0.539 & N/A & N/A & 0.437 & 0.539 \\
\hline & & HSA & 0.029 & N/A & N/A & 0.029 & 0.029 \\
\hline \multirow{2}{*}{ Giant cell } & Chitosan SRT & Integra & $<0.001$ & $<0.001$ & $<0.001$ & $<0.001$ & $<0.001$ \\
\hline & & HSA & $<0.001$ & $<0.001$ & $<0.001$ & $<0.001$ & $<0.001$ \\
\hline \multirow{2}{*}{$\begin{array}{l}\text { Invagination into } \\
\text { test material }\end{array}$} & Chitosan SRT & Integra & 0.566 & 0.566 & 0.566 & N/A & 0.566 \\
\hline & & HSA & $<0.001$ & $<0.001$ & $<0.001$ & N/A & $<0.001$ \\
\hline \multirow[t]{2}{*}{ Angiogenesis } & Chitosan SRT & Integra & N/A & 1.000 & 0.069 & 0.095 & N/A \\
\hline & & HSA & N/A & 0.292 & $<0.001$ & $<0.001$ & N/A \\
\hline
\end{tabular}

(N/A—not applicable).

Granuloma formation was detected in moderate amounts in both Integra and Chitosan SRT throughout the experiment (1.0-2.0 au). The number of granulomas was lower in the HSA samples (0.0-1.0 au). The number of giant cells was also generally low in samples of all three skin substitutes throughout the implantation period.

Tissue invagination into Chitosan SRT and Integra was observed by day 4 . The degree of invagination was almost complete by this time. The invagination into HSA samples only started at day 7 and was almost complete by day 21 (Figure 3).

\section{Discussion}

In this study we compared the biocompatibility of three different skin substitutes. Two of these substitutes (Integra 


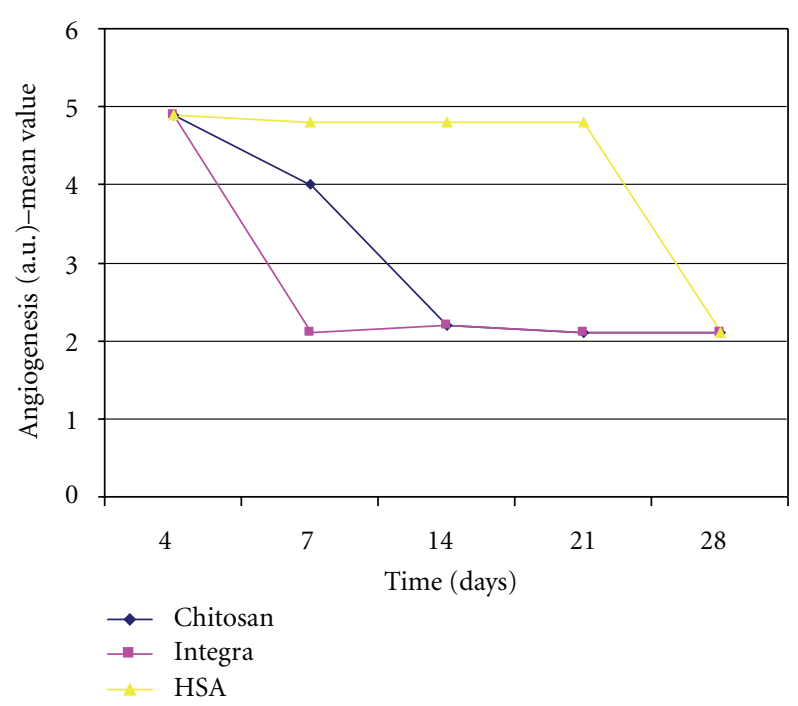

Figure 2: The level of angiogenesis (in mean arbitrary units) versus time for Chitosan SRT, Integra, and HSA.

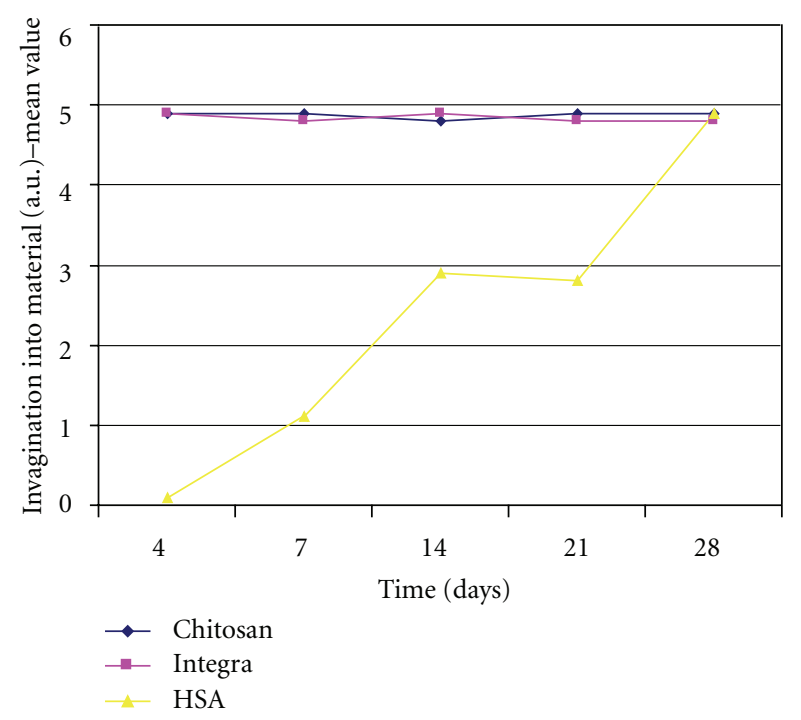

Figure 3: Level of invagination into the test material (in mean arbitrary units) versus time for Chitosan SRT, Integra, and human skin allograft (HSA).

and HSA) are already on the market and have been widely used. The third substitute, Chitosan SRT, is not available commercially. It is produced by the SIRIM Advanced Materials Research Centre, which is an organization involved in industrial research in Malaysia.

Chitosan is a cationic polysaccharide composed of glucosamine and $\mathrm{N}$-acetylglucosamine residues. It is relatively cheap and available in abundance. Chitosan is nontoxic, biocompatible, and has antibacterial, haemostatic and antitumoral properties [5-11].

Throughout the test period, Chitosan SRT did not induce an exaggerated inflammatory response. The number of acute inflammatory cells (Neutrophils/polymorphonuclear

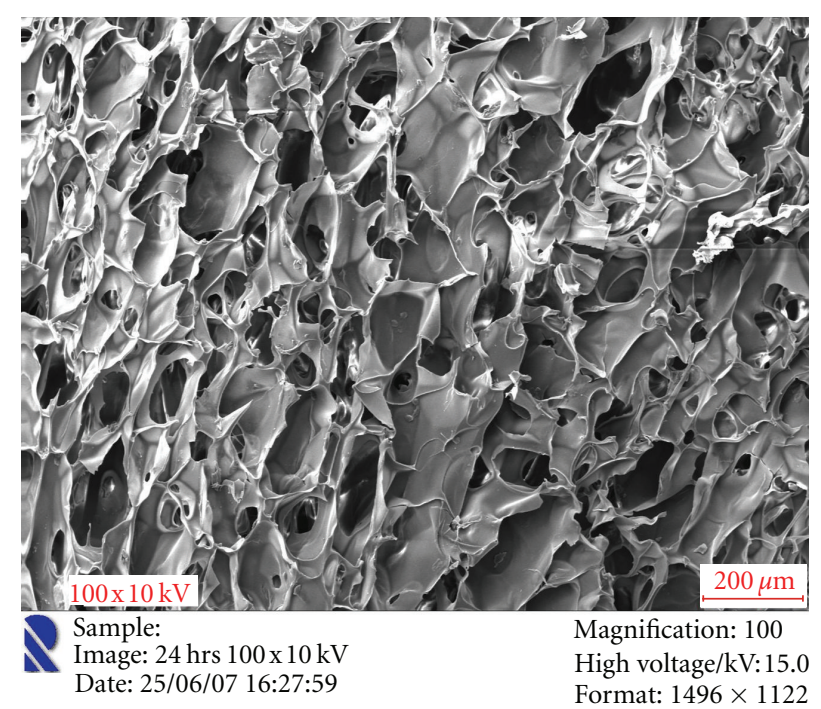

FigURE 4: Electron microscopy of the structure of Chitosan SRT and pore size.

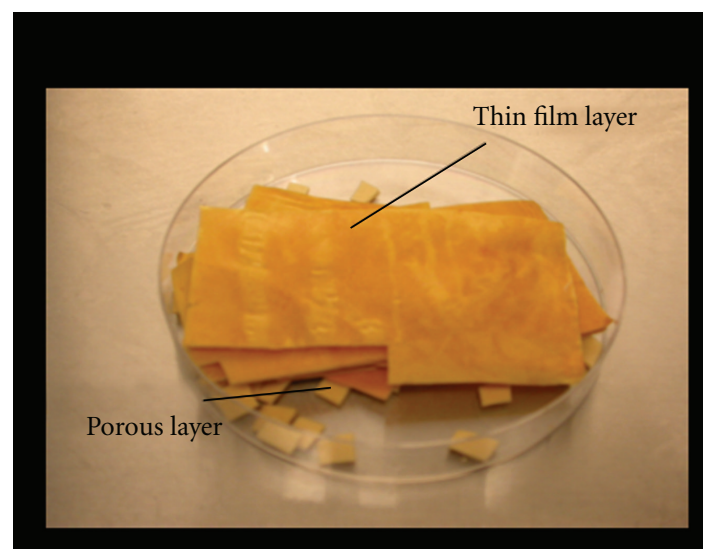

FIgURE 5: Photo of Chitosan SRT used in this study.

leukocytes, PMN) in the surrounding tissue in contact with the Chitosan SRT was demonstrated to be low. This may indicate an in vivo anti-inflammatory/antioxidant potential of the chitosan-based membranes. This could explain the low levels of acute inflammatory cells in the Chitosan SRT group. However, our study did show some amount of acute inflammation at days 4 through 7 . This could be the result of wounding rather than an effect of chitosan itself. After implantation of a medical device, the tissue will inevitably be traumatized by the implantation procedure, triggering an inflammatory response.

Proinflammatory cytokines that are released or activated by chitosan could also explain the low level of inflammatory response. Their roles in regulating immunological responses, hematopoietic development, and cell-to-cell communication, as well as host responses to infectious agents and inflammatory stimuli, have been widely investigated [19]. Under normal circumstances, cytokines are not detectable at low levels in body fluids or in tissues. Therefore, their 
activated levels of expression could indicate activation of cytokine pathways associated with inflammation. This could further explain the low levels of inflammation associated with chitosan.

The functional properties of chitosan and its derivatives are mainly dependent upon their molecular weight (MW) and the consequent ease of solubility in aqueous media [20]. The degree of deacetylation has also been shown to alter inflammatory reactivity [21]. The lower inflammatory reaction observed in some chitosan preparations may be due to the lower toxicity [22]. The Chitosan SRT that we used had a molecular weight $(\mathrm{Mv})$ of $6 \times 10^{5}$ daltons and $89 \%$ deacetylation.

Integra and Chitosan SRT induced similar patterns of inflammatory response. Integra had a higher number of inflammatory cells at day 14; however, the increase was not statistically significant. Integra has been shown to be biocompatible and inert [23]. This is because the "dermal" component is composed of highly purified type 1 bovine collagen that is of low antigenicity. King et al. [23] demonstrated that Integra only initiated a very minimal acute inflammatory reaction upon being vascularized.

Here we have shown that the Chitosan SRT used seems to induce very little inflammatory reaction in the surrounding tissue and is comparable to Integra.

The major sources of collagen currently used for biomedical applications are bovine or porcine skin or bovine or equine Achilles tendons. One disadvantage of these collagenbased biomaterials, which is a limiting factor for the widespread clinical application, is their mild immunogenicity imparted by the composition of the terminal region and the antigenic sites in the central helix. The immune response has been found to vary depending on the species from which collagen has been isolated, processing techniques used, and the site of implantation. Other concerns include the high cost of pure collagen, variable physicochemical properties and degradation patterns, and the risk of infectious disease transmission due to the allogenic or xenogenic origin of the material. With a chitosan-based biomaterial, most of these concerns will not be applicable.

HSA induced a higher level of inflammation compared to Integra and chitosan. HSA acts as a xenograft in the rat model. It also maintains its antigenicity due to the transplanted Langerhans cells in the epidermis. This could explain the higher levels of inflammation observed in the first 2 weeks compared to both Chitosan and Integra. However, the levels of inflammation were not persistently high or exaggerated.

During the implantation period, the three skin substitutes implanted did not recruit a large number of giant cells. Of the three, however, chitosan SRT seems to illicit the highest number. It had a mean of 0.9 au to 1.9 au for the first 21 days before reducing to 1.1 au at day 28 . This is compared to Integra and HSA which had a mean of 0.1 au during the same period $(P<0.001)$. Others have also demonstrated a slight foreign-body giant cell reaction obtained with chitosan when samples were implanted in the peritoneum of rats [24].

The rate of degradation of chitosan depends inversely on the degree of acetylation and crystallinity of the polymer
[25]. The longer it remains in vivo, the more likely it is to instigate a foreign-body response. The chitosan used in our study was $89 \%$ deacetylated; hence, we can expect some foreign-body reaction as its degradation would be slow.

There was intense angiogenesis occurring with all three skin substitutes tested at day 4 . This slowly decreased from day 7 for both Integra and Chitosan SRT. In the HSA group, the intensity of angiogenesis remained high for 21 days before reducing. Angiogenesis was similar in all three groups by day 28 .

Chitosan has been shown to physiologically stimulate the tissue repair process and favors angiogenesis [26]. However, low molecular weight chitosan $(100 \mathrm{Ag})$ has proven to be a potent inhibitor of angiogenesis. This role of chitosan is currently being explored in antitumor research [20]. Chitosan in the form of a scaffold has been shown to carry angiogenic activity when implanted [27]. Hence, the use of chitosan, which can act as a promoter of angiogenesis, as an artificial dermis is logical.

The structures of Chitosan SRT and Integra used explain the pattern of tissue invasion into the material. Chitosan SRT and Integra had complete tissue invasion by day 4 . Both Chitosan SRT and Integra are in the form of a bilayer skin template. The inner layer was meant to act as a scaffold for tissue invasion. As the growth template is already present, the rapid rate of tissue invasion is expected. Mi et al. [22] also demonstrated that the sponge-like architecture of chitosan could provide an adsorption capacity for fluids, draining the wound by capillary action and enhancing tissue regeneration.

The in vivo biological acceptance of an implanted biomaterial always involves inflammation and wound healing, without which the body would only tolerate the biomaterial instead of incorporating it into a functional and longterm association. We have demonstrated that all three test materials examined here are biocompatible, as they did not show an intense or prolonged acute inflammatory phase.

\section{Conclusion}

We have demonstrated that Chitosan SRT, Integra, and HSA did not illicit intense and prolonged inflammatory reactions. None of the three test materials caused an adverse foreign body reaction, and all were tolerated well in this animal model. The three skin substitutes have been shown to be biocompatible.

Chitosan SRT used is also shown to have similar inflammatory, angiogenesis, and tissue ingrowth responses to Integra. Based on this study, Chitosan SRT could serve as an alternative to Integra and HSA as a skin substitute.

\section{Acknowledgments}

The authors would like to acknowledge the Intensification of Research in Priority Area Programme (IRPA, Grant no. 305/PPSP/6112256), Ministry of Science, Technology and Innovation (MOSTI), Malaysia, for providing the funds to make this study come to reality. We would also like to 
acknowledge Dr. Ismail Zainol from AMREC for preparing the Chitosan SRT.

\section{References}

[1] R. G. Tompkins and J. E. Burke, "Alternative wound coverings," in Total Burn Care, D. Herndon, Ed., pp. 164-172, WB Saunders, Philadelphia, Pa, USA, 1st edition, 1996.

[2] B. A. Pruitt and N. S. Levine, "Characteristics and uses of biologic dressing and skin substitutes," Archives of Surgery, vol. 119, no. 3, pp. 312-322, 1984.

[3] T. J. Phillips, "New skin for old: developments in biological skin substitutes," Archives of Dermatology, vol. 134, no. 3, pp. 344-349, 1998.

[4] G. G. Gallico, "Biologic skin substitutes," Clinics in Plastic Surgery, vol. 17, no. 3, pp. 519-526, 1990.

[5] K. Nishimura, S. Nishimura, N. Nishi, I. Saiki, S. Tokura, and I. Azuma, "Immunological activity of chitin and its derivatives," Vaccine, vol. 2, no. 1, pp. 93-99, 1984.

[6] Y. Okamoto, S. Minami, A. Matsuhashi et al., "Polymeric Nacetyl-D-glucosamine (chitin) induces histionic activation in dogs," The Journal of Veterinary Medical Science, vol. 55, no. 5, pp. 739-742, 1993.

[7] E. Khor and L. Y. Lim, "Implantable applications of chitin and chitosan," Biomaterials, vol. 24, no. 13, pp. 2339-2349, 2003.

[8] T. Mori, M. Okumura, M. Matsuura et al., "Effects of chitin and its derivatives on the proliferation and cytokine production of fibroblasts in vitro," Biomaterials, vol. 18, no. 13, pp. 947-951, 1997.

[9] A. K. Singla and M. Chawla, "Chitosan: some pharmaceutical and biological aspects_-an update," Journal of Pharmacy and Pharmacology, vol. 53, no. 8, pp. 1047-1067, 2001.

[10] T. Tanigawa, Y. Tanaka, H. Sashiwa, H. Saimoto, and Y. Shigemasa, "Various biological effects of chitin derivatives," in Advances in Chitin and Chitosan, C. J. Brine, P. A. Sandford, and J. P. Zikakis, Eds., pp. 206-215, Elsevier, New York, NY, USA, 1992.

[11] S. Tokura, K. Ueno, S. Miyazaki, and N. Nishi, "Molecular weight dependent antimicrobial activity by chitosan," in Macromol Symp, vol. 120, pp. 1-9, 1997.

[12] B. Krajewska, "Membrane-based processes performed with use of chitin/chitosan materials," Separation and Purification Technology, vol. 41, no. 3, pp. 305-312, 2005.

[13] I. V. Yannas, J. F. Burke, P. L. Gordon, C. Huang, and R. H. Rubenstein, "Design of an artificial skin. II. Control of chemical composition," Journal of Biomedical Materials Research, vol. 14, no. 2, pp. 107-131, 1980.

[14] I. Jones, L. Currie, and R. Martin, "A guide to biological skin substitutes," British Journal of Plastic Surgery, vol. 55, no. 3, pp. 185-193, 2002.

[15] R. L. McCauley, “The skin bank," in Total Burn Care, D. N. Herndon, Ed., pp. 159-163, Saunders, London, UK, 1996.

[16] L. Hussmann, R. C. Russell, J. O. Kucan, D. Hebebrand, T. Bradley, and H. U. Steinau, "Use of glycerolized human allografts as temporary (and permanent) cover in adults and children," Burns, vol. 20, no. 1, pp. S61-S66, 1994.

[17] I. Zainol, A. S. Halim, S. M. Ghani et al., "Preparation and characterization of bilayer chitosan membrane as skin renerating template," Malaysian Journal of Microscopy, vol. 14, pp. 24-29, 2008.

[18] I. Zainol, A. S. Halim, Z. Ujang, and K. Hashim, "A method for producing skin regenerating template," Patent Pending, no. PI 20072205, 2007.
[19] C. K. Lim and A. S. Halim, "In vitro models in biocompatibility assessment for biomedical-grade chitosan derivatives in wound management," International Journal of Molecular Sciences, vol. 10, no. 3, pp. 1300-1313, 2009.

[20] K. V. H. Prashanth and R. N. Tharanathan, "Depolymerized products of chitosan as potent inhibitors of tumor-induced angiogenesis," Biochimica et Biophysica Acta, vol. 1722, no. 1, pp. 22-29, 2005.

[21] C. J. Park, N. P. Gabrielson, D. W. Pack, R. D. Jamison, and A. J. W. Johnson, "The effect of chitosan on the migration of neutrophil-like HL60 cells, mediated by IL-8," Biomaterials, vol. 30, no. 4, pp. 436-444, 2009.

[22] F. L. Mi, Y. C. Tan, H. F. Liang, and H. W. Sung, "In vivo biocompatibility and degradability of a novel injectablechitosan-based implant," Biomaterials, vol. 23, no. 1, pp. 181191, 2002.

[23] W. W. K. King, P. K. Lam, C. T. Liew, W. S. Ho, and K. C. Li, "Evaluation of artificial skin (Integra) in a rodent model," Burns, vol. 23, supplement 1, pp. S30-S32, 1997.

[24] Z. L. Zhang, S. W. Xu, and X. L. Zhou, "Preventive effects of chitosan on peritoneal adhesion in rats," World Journal of Gastroenterology, vol. 12, no. 28, pp. 4572-4577, 2006.

[25] C. Shi, Y. Zhu, X. Ran, M. Wang, Y. Su, and T. Cheng, "Therapeutic potential of chitosan and its derivatives in regenerative medicine," Journal of Surgical Research, vol. 133, no. 2, pp. 185-192, 2006.

[26] G. Biagini, A. Pugnaloni, A. Damadei, A. Bertani, A. Belligolli, and V. R. B. Muzzarelli, "Morphological study of the capsular organization around tissue expanders coated with $\mathrm{N}$ carboxybutyl chitosan," Biomaterials, vol. 12, no. 3, pp. 287291, 1991.

[27] P. J. VandeVord, H. W. T. Matthew, and S. P. DeSilva, "Evaluation of the biocompatibility of chitosan in mice," Journal of Biomedical Research B, vol. 59, no. 3, pp. 585-590, 2001. 

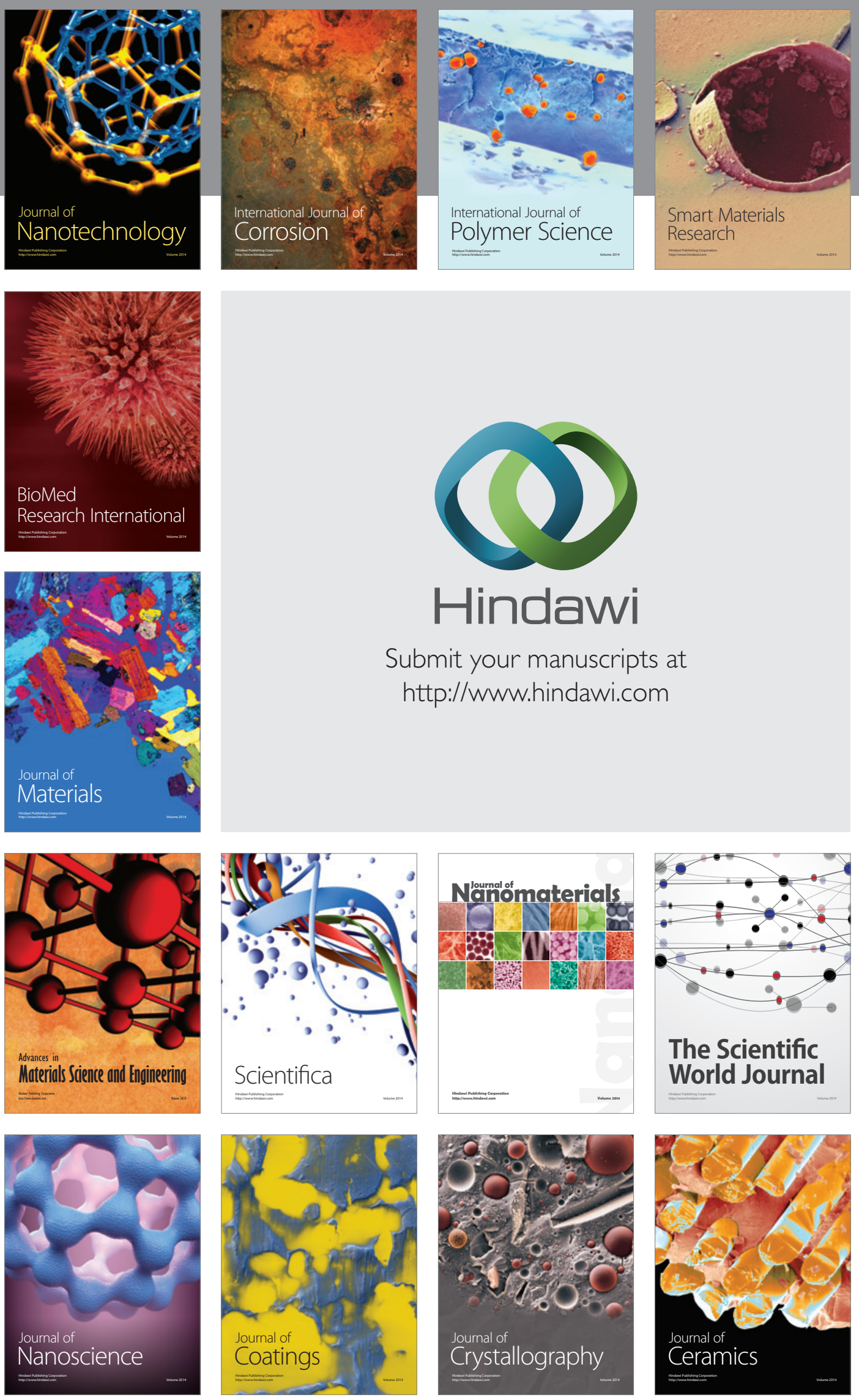

The Scientific World Journal

Submit your manuscripts at

http://www.hindawi.com

\section{World Journal}

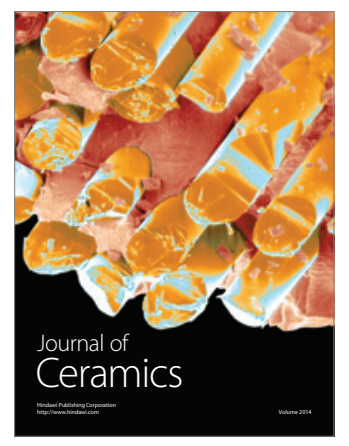

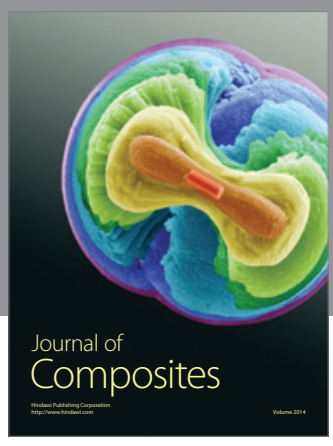
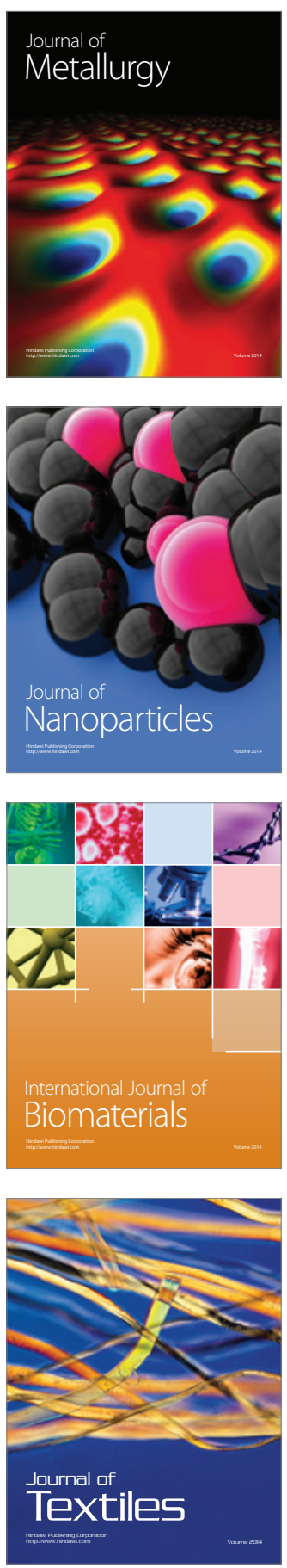